\section{P149 Fruit and Vegetable Consumption of Michigan Youth and Adults: Locally Implemented SNAP-Ed Programs with Aggregated Impact}

Marci Scott, PhD,RDN, Michigan Fitness Foundation; Kerri Vasold, PhD, ACSM-PAPHS, Michigan Fitness Foundation; Lila Gutuskey, PhD, lgutuskey@michiganfitness.org, Michigan Fitness Foundation, 1213 Center St, Lansing, MI 48906

Objective: Supplemental Nutrition Assistance Program Education (SNAP-Ed) at the Michigan Fitness Foundation is a collaboration of local and regional organizations whose work focuses on improving the health of Michigan's most vulnerable citizens. While organizations select and implement locally relevant programming, evaluation plans are designed to report common fruit and vegetable consumption measures aligned with the SNAP-Ed Evaluation Framework.

Use of Theory or Research: Aligned with the social ecological framework, SNAP-Ed works to address factors of health behavior across multiple levels of influence. While there is broad literature and understanding on the factors that influence healthy eating, programs can do the most good by addressing the local needs of the communities they serve.

Target Audience: Youth (grades 6-12) and adults (aged $18+)$ who received SNAP-Ed programming that included evidence-based nutrition education interventions based on local needs assessments.

Program Description: Design a common program evaluation that could be applied to a variety of direct education interventions to support local-level objectives and be aggregated to demonstrate state-level outcomes.

Evaluation Methods: Participants received a dietary questionnaire pre- and post-intervention. Participant data were matched based on demographic indicators and data were aggregated at the state-level. Descriptive statistics were calculated for all variables of interest, and Wilcoxon Signed Rank tests were used to compare differences pre- and post-intervention for fruit and vegetable consumption.

Results: Participants included 1512 youth and 647 adults from SNAP-Ed programs delivered by 20 community organizations across Michigan. A statistically significant increase was found for youth frequency of fruit consumption (pre: $1.62 \pm 1.62$; post: $1.75 \pm 1.69 ; P=.022$ ) and of vegetable consumption (pre: $1.70 \pm 1.97$; post: $1.88 \pm 2.17 ; \quad P=.002)$. Results were similar for adults with a statistically significant increase in frequency of fruit consumption (pre: $3.17 \pm 1.23$; post: $3.66 \pm 1.19$; $P<.001$ ) and of vegetable consumption (pre: $3.35 \pm 1.14$; post: $3.80 \pm 1.14 ; P<.001)$.

Conclusions: Implementation of locally relevant SNAPEd programming selected by community organizations can positively impact fruit and vegetable consumption. Future research should explore characteristics across locally relevant programs to identify key implementation variables for greatest impact.

Funding: Supplemental Nutrition Assistance Program Education.

\section{P150 Development of an 8-Week Early Childhood Nutrition Education Intervention for Children with Autism Spectrum Disorder and their Parents}

Whitney Van Arsdale, BS, wvanarsdale@usf.edu, University of South Florida, College of Public Health, 13201 Bruce B. Downs Blvd, MDC 56, Tampa, FL, 33612; Heewon Gray, $P h D, R D N$, University of South Florida, College of Public Health; Acadia Buro, MS, University of South Florida, College of Public Health

Objective: Children with autism spectrum disorder (ASD) experience more problematic mealtime behaviors compared to neurotypical children. The prevalence of overweight/obesity is also higher in children with ASD. The aim of this study was to develop a comprehensive nutrition education intervention program to be used by Early Intervention providers who work with children with ASD and their parents.

Use of Theory or Research: The nutrition education manual for early intervention providers was developed based on the 2019 Academy of Nutrition and Dietetics Pediatric Nutritional Care Manual and other evidencebased autism-specific feeding strategies, in conjunction with the social cognitive theory for behavioral change.

Target Audience: Early intervention providers who work with children with ASD ages 0-3 years and their parents.

Program Description: The Autism Eats program with eight 20-minute weekly lessons addresses: (1) Feeding milestones, (2) Exploring taste/flavor/texture, (3) Mealtime routines/schedules, (4) Balanced eating/nutrition, (5) Strategies for introducing new foods, (6) Dining-out and beverages, (7) Fill-and-Full, and (8) Maintaining healthy nutrition.

Evaluation Methods: A Qualtrics survey was used to measure providers' perceived feasibility and acceptability of the nutrition education intervention. The survey consisted of 7 Likert scale questions (from "strongly disagree" to "strongly agree") and 3 open-ended questions for each lesson. Four health psychology experts who work as providers to children with ASD reviewed the manual and completed the survey.

Results: The mean scores were greater than 4 (of a possible 5) points for all questions, including preparation time, clarity, activity relevance, background information, feasibility, and overall relevance to the goal, except for the question on estimated ability to complete the weekly activity with the parent/child within the 20-minute time frame, which had a mean of 3.9 (ranged 2.5-5).

Conclusions: The Autism Eats program provides meaningful information and activities relevant to addressing feeding behavioral problems and autism-specific nutritional concerns. Minor revisions are needed to adjust the length of the activities to comply with the pre-determined 20-minute time slot.

Funding: University of South Florida Women's Health Collaborative Betty Castor Grant. 